\title{
Stationary phantoms and grating induction with oblique inducing gratings: Implications for different mechanisms underlying the two phenomena
}

\author{
JAMES M. BROWN \\ University of Georgia, Athens, Georgia \\ JIRO GYOBA \\ Tohoku University, Sendai, Japan \\ and \\ JAMES G. MAY \\ University of New Orleans, New Orleans, Louisiana
}

\begin{abstract}
The visibility of stationary visual phantoms and the grating induction (GI) effect were concurrently analyzed with both black and gray inspection areas (IA) using the same subjects with counterbalanced orders of measurements. Oblique inducing gratings were employed in order to compare the visibility of obliquely aligned and vertically misaligned appearances between the two phenomena. Aligned and misaligned phantom responses with a black IA were similar, whereas overall phantom visibility was severely suppressed when the IA was gray. In contrast, misaligned GI dominated with a gray IA, whereas aligned and misaligned GI responses were similar with a black IA. Phantoms appear to be related to visual mechanisms' selectively utilizing relative luminance information between the inducing grating and IA in a manner consistent with more global figural characteristicsof the display (e.g., modal and amodal completion). On the other hand, GI may be predominantly due to locally operating brightness/contrast mechanisms.
\end{abstract}

The visual phantom illusion is induced by a vertical grating that is interrupted by a horizontal black inspection area (IA). A phantom grating is perceived to continue across the IA, dimly but unmistakably. Tynan and Sekuler (1975) originally reported the illusion using moving inducing gratings. Later, phantoms were also found to be produced by flickering (Genter \& Weisstein, 1981) and stationary gratings (Gyoba, 1983). On the other hand, using a similar display, McCourt (1982) discovered the grating induction (GI) effect in which a vertical inducing grating produces the appearance of an opposite phase grating within a homogeneous gray IA. The question of whether or not phantoms and GI are produced by the same mechanisms has been discussed in numerous reports (Foley \& McCourt, 1985; Gyoba, 1994a; May, Brown, \& Roberts, 1999; McCourt, 1994; Sakurai \& Gyoba, 1985). These two phenomena share common characteristics, but also have distinctive properties, as described below.

McCourt (1994) summarized a number of interesting similarities between phantoms and GI as follows. Both effects survive dichoptic presentation of the inducing grating and the IA, increase in strength with decreasing

Correspondence concerning this article should be addressed to J. M. Brown, Department of Psychology, University of Georgia, Athens, GA 30602-3013 (e-mail: jmbrown@ arches.uga.edu). inducing grating spatial frequency, occur more strongly with sine wave than with square wave gratings, and require a temporal integration period up to $400 \mathrm{msec}$ to achieve full strength. Moreover, on the basis of psychophysical data obtained by a pointwise brightness matching paradigm, McCourt (1994) pointed out that the illusory grating is not "in phase" with the inducing grating as is commonly assumed, but is actually in opposite phase (180 ${ }^{\circ}$ out of phase) with it. Therefore, McCourt (1994) suggested that stationary phantoms may be a manifestation of the same processes underlying GI. Indeed, this raised the possibility that subjects instructed to indicate the presence of illusory phantoms might commonly report the presence of grating induction instead.

Recently, this question was addressed with a simultaneous brightness matching technique in which subjects were instructed regarding the differences between phantoms (illusions that comprise brightness differences in phase with inducing stimuli) and grating induction (illusions that comprise brightness differences out of phase with inducing stimuli) and were asked to respond by indicating the presence of phantoms or GI after a brief inspection period (May et al., 1999). This technique required a fixation point, which has been shown to reduce phantom visibility, but even under these conditions, subjects reliably reported the presence of real and illusory in-phase grat- 
ings, indicating that they could reliably use the response categories.

Regarding distinctive properties, Sakurai and Gyoba (1985) revealed that the luminance of the IA is a determinant of the two phenomena. Stationary phantom visibility is maximal when the IA luminance is close to the maximum or to the minimum level of the inducing grating, and between these levels, especially at mean luminance, the grating induction effect is dominantly perceived (for a review of similar findings for moving and flickering phantoms see Maguire \& Brown, 1987). Moreover, Gyoba (1994a) found that stationary phantoms are perceived with only a low luminance (less than $80 \mathrm{~cd} / \mathrm{m}^{2}$ ) inducing grating, whereas the magnitude of GI increases as the luminance of the inducing grating increases into photopic levels (McCourt, 1990).

Another important property in discussing the mechanisms underlying phantoms and GI is the differences in the figural nature of their appearance. For example, when patterned stripes make up the inducing grating, moving phantoms are produced that are themselves patterned (Weisstein, Maguire, \& Berbaum, 1977). Phantom visibility is also affected by the overall figure/ground relations in the inducing display. Brown and Weisstein (1991) found that moving phantom visibility was reduced when figure/ground information remote from the IA indicated that it was in front of the parts of the inducing display that completed as phantoms. The phantom phenomenon has also been shown to produce a phantom context effect (Brown \& Weisstein, 1988) in which orientation discrimination is better in the areas of the IA perceived as phantoms compared with those areas not seen as phantoms, or when no phantoms are seen in the IA at all. Recently, Gyoba (1994b) found that when a stationary inducing grating has an oblique orientation, "aligned phantoms" are perceived obliquely along the orientation of inducing grating, whereas "misaligned phantoms" are also produced vertically by connecting the nearest bars between the top and bottom sections of the inducing grating. By contrast, such flexible characteristics have not been reported for GI. As Zaidi (1989) demonstrated, the perceived spatial frequency and orientation of GI are different from those of the inducing grating. He suggested that the local edge effects are primarily responsible for GI and that global properties of inducing gratings are less important.

In the present experiment, we used the simultaneous brightness comparison task and asked subjects to make either phantom or GI judgments under various conditions so that direct comparisons between the two phenomena could be made. The purpose of the present study was to make a concurrent analysis of phantom and GI visibility with both black and gray IAs using the same subjects with counterbalanced orders of measurements. Oblique inducing gratings are employed in order to compare the visibility of aligned and misaligned appearances between the two phenomena. Such analyses enable us to investi- gate precisely whether phantoms and GI really have (1) a unique condition for optimal viewing, and (2) different figural characteristics. The results of the present study reveal important information as to whether the two phenomena can be accounted for by the same mechanisms, and whether they are alternate forms of the same illusion, or are different illusions arising from different mechanisms.

Considering the differences between GI and phantoms and the flexibility of phantoms related to organizational factors discussed above, it was important to carefully inform the subjects about the perceptual characteristics that distinguish GI and phantoms. The subjects were first given general instructions using vertical inducing stimuli. These were followed by specific instructions concerning the possibility of aligned and misaligned GI and phantoms using an oblique inducing grating. Through the combination of instructions and having each observer make both GI and phantom judgments, it was expected that criterion related variability would be reduced.

Testing both phenomena with a black and a gray IA allowed us to make specific predictions about phantom and GI visibility. On the basis of previous phantom studies (Brown, 1985; Sakurai \& Gyoba, 1985), we predicted optimal phantom visibility with a black IA and little if any with a gray one. On the basis of previous GI studies (Foley \& McCourt, 1985; McCourt, 1982, 1994), we predicted optimal GI visibility with a gray IA. We were less certain about GI visibility with a black IA, although past results (Foley \& McCourt, 1985; McCourt, 1982, 1994) have suggested a considerably reduced effect should occur.

Using an oblique inducing grating created the possibility for GI and phantoms to appear in both aligned and misaligned orientations. This variable also allowed us to specifically test whether phantoms and GI are the same phenomenon. If GI is a lower level, local, contrast-like induction effect with little perceptual flexibility or figural characteristics, then under optimal conditions (i.e., with a gray IA) GI should appear more in the misaligned than in the aligned orientation. This prediction was based on the GI findings of Zaidi (1989) and the fact that the shortest distance across the IA is in the misaligned direction. Although the figural continuity of the inducing grating is in the aligned orientation in our displays, this orientation would require the induction to occur over a longer distance. However, if figural qualities often noted in phantom perception are also active in GI perception, aligned and misaligned GI should appear equally often with a gray IA. How orientation might effect GI visibility with a black IA was less clear. As noted, GI magnitude with a black IA is considerably less than with a gray IA (McCourt, 1982, 1994). Due to the uncertainty about GI visibility with a black IA, no specific predictions were made about the influence of orientation in this condition. How would orientation influence phantom visibility? On the basis of the figural fidelity and organizational flexibility of phantoms and on Gyoba's (1994b) findings using a low spatial frequency (e.g., $1 \mathrm{cpd}$ ) oblique inducing 
grating and a black IA, aligned and misaligned phantom visibility should be nearly the same with a black IA. As noted above, few if any phantoms would be expected with a gray IA.

\section{METHOD}

\section{Subjects}

Forty introductory psychology students from the University of Georgia participated for course credit. All subjects were given an eye exam (Orthorater) and had normal (20/20) or corrected-tonormal vision.

\section{Stimuli and Apparatus}

The stimuli were created and presented with a computer controlled Data Translation 2862 Frame Grabber output to an NEC DM-2000P RGB monitor. A matte black reduction screen was placed in front of the monitor, producing a rectangular viewing region where, from the $149-\mathrm{cm}$ viewing distance, the inducing grating subtended $2.9^{\circ}(\mathrm{h}) \times 10^{\circ}(\mathrm{w})$ above and below a $1.15^{\circ}(\mathrm{h}) \times 10^{\circ}$ (w) sized IA. The 1-cpd sine-wave inducing grating had a contrast of 0.60 and a mean luminance of $11.9 \mathrm{~cd} / \mathrm{m}^{2}$. When the IA was black, it had a luminance equal to the minimum luminance value of the inducing grating $\left(4.8 \mathrm{~cd} / \mathrm{m}^{2}\right)$, and when it was gray it had a luminance of $11.9 \mathrm{~cd} / \mathrm{m}^{2}$. A small $\left(7 \times 7 \mathrm{in}\right.$.), $\operatorname{dim}\left(8.0 \mathrm{~cd} / \mathrm{m}^{2}\right)$ fixation point was always present. During the general introduction to the phenomena, the inducing grating was presented in a vertical orientation. During the subsequent specific instructions and the actual experiment, the inducing grating was oriented $46^{\circ}$ from vertical (see Figure 1). Viewing was binocular from a chinrest.

\section{Procedure}

Subjects were tested one at a time, seated in a dark room with the only illumination coming from the monitor. After dark adaptation for $5 \mathrm{~min}$, subjects were introduced to the phenomena of visual phantoms and GI. The order in which the phenomena were introduced (GI or phantoms first) and subsequently tested were counterbalanced across observers. The general instructions focused on the distinguishing characteristics of each phenomenon, and the specific instructions focused on their appearance with an oblique inducing grating (see below).
During the experiment, a stimulus was presented on each trial for $30 \mathrm{sec}$ with a 25 -sec intertrial interval. Between trials, the screen was blank with a mean luminance of $11.9 \mathrm{~cd} / \mathrm{m}^{2}$. Trials alternated between having a black and a gray IA. IA order was counterbalanced so that an equal number of subjects saw either the black or the gray IA first during the experimental trials. For each half of the experiment, subjects were instructed to respond to only one of the phenomena, with the order of phenomena counterbalanced. Each half of the experiment consisted of 12 trials, 6 with each IA. The first two trials with each IA for each half of the experiment were excluded from the analysis. The percentage of total viewing time was calculated from the remaining 4 trials for a total viewing time of 2 min per condition.

Introductory instructions. GI and phantoms were defined with both gray and black IAs, and local brightness characteristics in the IA were highlighted. GI was defined as being when the IA looks darker between the light and lighter between the dark grating stripes. It was noted the out-of-phase grating might only be visible part of the way into the IA, fading out in the middle. Subjects were instructed that, whether the out-of-phase grating appeared all the way across the IA or faded in the middle, they were seeing GI. Phantoms were defined as being when any of the black inducing grating stripes appeared complete in front of the IA in phase with the inducing grating (i.e., darker between the dark stripes and lighter between the light stripes). This perceptual completion and possible depth characteristics were noted. It was pointed out that the perception of GI and phantoms could fluctuate, sometimes being visible and sometimes not. The illusory nature of both phenomena were demonstrated by showing how the perception of them was eliminated when the border between the IA and the inducing grating was momentarily covered. Subjects were informed that the experiment consisted of two parts and that specific instructions would precede each part.

Experimental instructions. As subjects transitioned from one part to the next-for example, from phantom judgments in part one to GI judgments in part two-the importance of making a distinction between phantom and GI judgments was stressed. It was pointed out that, during the present part, it did not matter whether they saw the effect they had just made judgments about in the previous part. They were instructed to make their judgments for the current part on the basis of the description in the introduction and before that part.
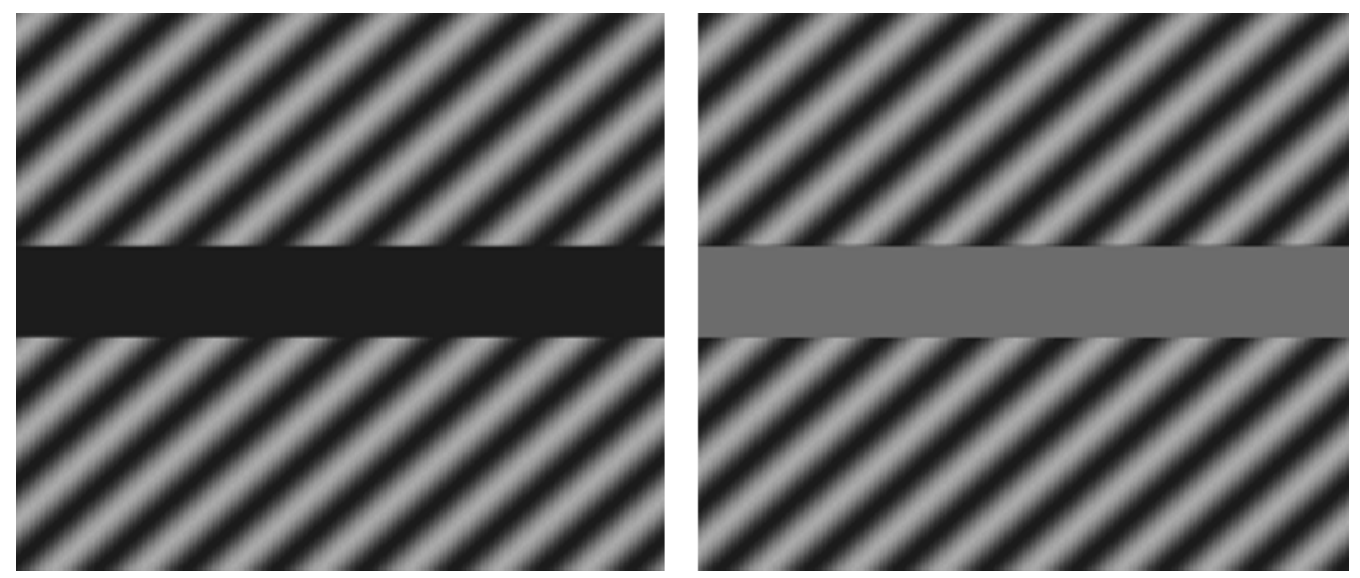

Figure 1. Oblique inducing grating with a black (left) and gray (right) IA. Phantoms and GI may appear aligned or misaligned (see text for details). 
During experimental instructions, a diagonal inducing grating was used with an IA that was either black or gray. The possibility that the phenomena currently being judged could be seen to occur aligned (obliquely) or misaligned (vertically) was pointed out, and the appropriate response keys were assigned. Subjects were then instructed that they would be making their judgments with both black and gray IAs. It was stressed that whether or not they saw the phenomenon was not important, but that it was important to press the appropriate response key (left arrow for aligned or right arrow for misaligned) only when they saw that it was occurring in that direction. If they saw nothing, they were to withhold a keypress.

\section{RESULTS}

A 2 (judgment: phantoms and GI) $\times 2$ (IA: black and gray) $\times 2$ (orientation: aligned and misaligned) analysis of variance (ANOVA) was conducted on the mean percentage of total viewing time. The analysis revealed a significant main effect of judgment $[F(1,39)=12.35, p<.001]$, indicating that overall, GI visibility was greater than phantom visibility. The reason for this was the extremely poor phantom visibility with a gray IA. The significant main effect of IA $[F(1,39)=8.88, p<.005]$ and significant judgment $\times$ IA interaction $[F(1,39)=25.11, p<.0001](\mathrm{de}-$ picted in Figure 2) indicate that phantom and GI visibility were affected differently, depending on whether the IA was black or gray. A Tukey's pairwise probe of the interaction showed that, as predicted, phantom visibility was significantly $(p<.05)$ greater with a black $(25 \%)$ than with a gray $(9 \%)$ IA. However, GI visibility was little affected by the lightness of the IA (black $=21 \%$, gray $=24 \%$ ).
The main effect of orientation and any interactions with this factor were not significant in the ANOVA. With little phantom visibility expected with a gray IA and no effect of orientation expected for phantoms with a black IA, it is not surprising that orientation was not significant in the ANOVA. We predicted that only one condition (GI with a gray IA) would show an influence of orientation, with greater visibility in the misaligned orientation. A planned contrast (a $t$ test using $\mathrm{MS}_{\mathrm{error}}$ as the variance estimate and evaluating $t$ on $d f_{\text {error }}$ degrees of freedom; Howell, 1987) on GI with a gray IA showed that GI visibility was significantly greater for the misaligned $(28.88 \%)$ than the aligned $(19.65 \%)$ orientation $\left(t_{39}=2.07, p<\right.$ $.025)$. Thus, as predicted, there were no differences in aligned and misaligned phantom visibility with a black IA, and there was greater misaligned than aligned GI visibility with a gray IA.

\section{DISCUSSION}

The present results for phantom judgments with black IAs are in agreement with the results of Gyoba (1994b). No difference was found between aligned and misaligned judgments with 1-cpd oblique inducing gratings in either that study or in the present study, but there was an overall difference between the two studies in the percentage of total viewing time during which phantoms were visible. Gyoba (1994b) found about $40 \%$, whereas the present study reported only about $25 \%$. Numerous differences in methodology (inducing grating contrast or luminance,

\section{VISUAL PHANTOMS GRATING INDUCTION}
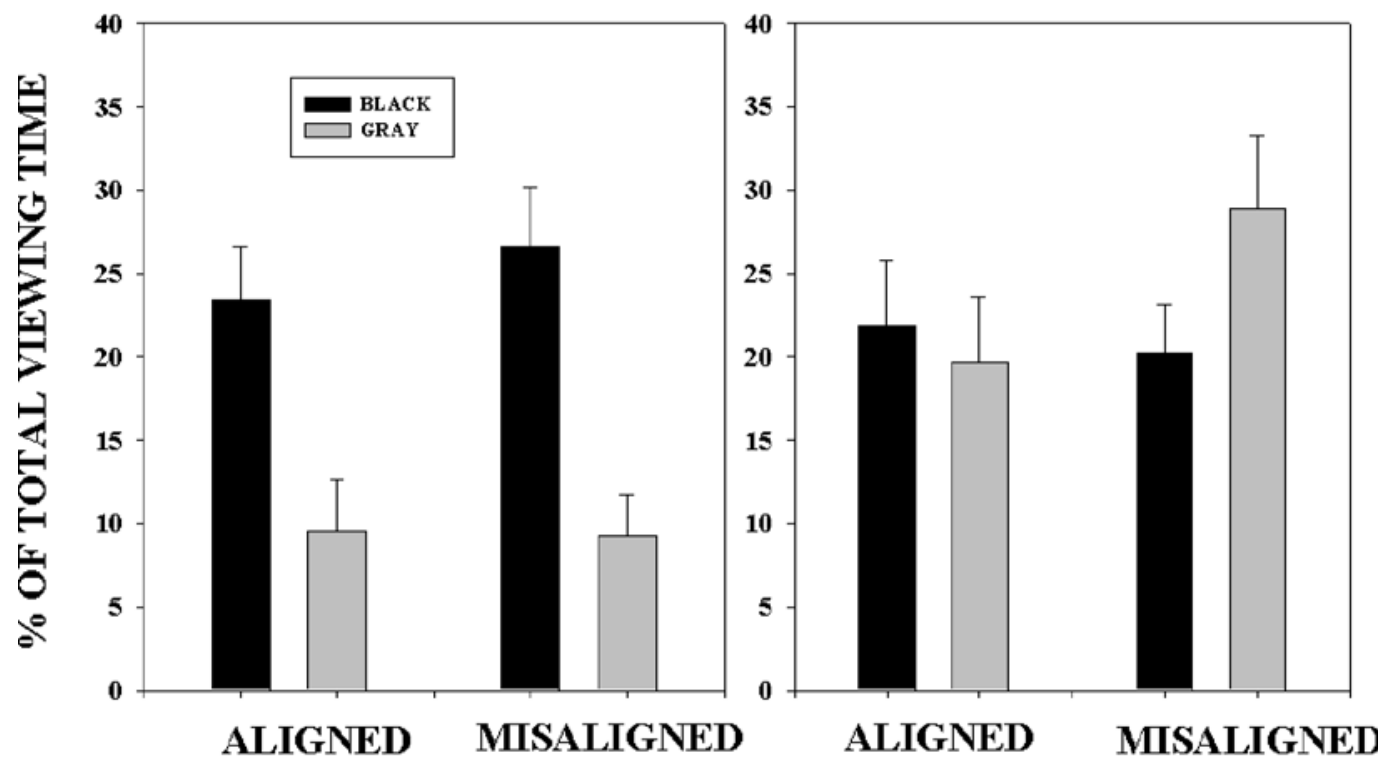

\section{JUDGMENT DIRECTION}

Figure 2. Percentage of total viewing time phantoms (left panel) and GI (right panel) were visible in aligned (oblique) and misaligned (vertical) orientations as a function of IA luminance. 
IA height, and subject differences) could have accounted for this difference. The finding that phantom visibility was best when the IA was black and was quite poor with gray IAs is also commensurate with previous findings (Brown, 1985; Gyoba, 1994a; Sakurai \& Gyoba, 1985).

In contrast to the strong IA luminance effect found with phantoms, GI visibility was quite similar for both gray and black IAs. Although this finding seems inconsistent with studies that have assessed GI visibility by using a cancellation technique (Foley \& McCourt, 1985; McCourt, 1982), it is in agreement with McCourt's (1994) most recent findings resulting from a pointwise brightness matching technique. As McCourt (1994) noted, "the contrast matching procedure involves a judgment of the contrast of a spatially extended grating, whereas the pointwise matching procedure requires strictly local brightness judgments" (p. 1614). It may be that our instructions focusing on the brightness differences within the IA between the light and dark inducing grating stripes produced more "localized" judgments, which contributed to the similar GI visibility with the gray and black IAs.

One unique finding of the present study was the differences in the aligned and misaligned results for each phenomenon. Although the visibility in these two categories did not differ within an IA condition for phantoms, more misaligned GI was observed in the gray IA condition. With the black IA, no significant differences between aligned and misaligned responses were observed. Thus, under the optimal conditions for perceiving GI (gray IA), misaligned responses were more frequent; under less favorable conditions (black IA), no difference was noted.

To summarize these findings, IA luminance is the most critical factor in determining whether phantoms or GI predominate. With a gray IA, phantom visibility was poor and GI was frequent. With a black IA, both phantom and GI visibility were frequent. This influence of IA luminance is well documented for stationary (Sakurai \& Gyoba, 1985), moving (Brown, 1985), and flickering phantoms (Brown \& Weisstein, 1985). The primary influence of IA luminance is to modify the edge information between the inducing grating and the IA. This edge information creates different possibilities as far as the perceptual organization of the IA relative to the inducing grating stripes. In addition, this edge information can be interpreted differently depending on whether local brightness/contrast judgments are being made about the IA or whether the perceiver is making judgments about global surface properties that involve modal and amodal completion and depth. Global organizational properties appear to play little part in local, brightness/contrast GI judgments. Thus, whether the IA is gray or black has little impact on whether GI is noted. For phantoms, IA luminance does influence its representation as a surface (Nakayama, He, \& Shimojo, 1995). A gray IA creates strong interposition cues so that the relatability (Kellman \& Shipley, 1991) of the inducing grating edges at the intersection of the IA favors the completion of the stripes amodally behind the IA, effec- tively eliminating the possibility that these edges will complete modally in front of it. With a black IA, there is ambiguity as to whether the black grating stripes complete modally or amodally relative to the IA. This ambiguity creates the possibility for phantoms. Thus, the way IA luminance influences GI and phantom visibility differently indicates an ability to shift focus between local brightness/contrast GI judgments and more global figure/ ground judgments using the same stimulus.

One perspective on the multistable aspect of a phantom/ GI inducing display is from Grossberg's FACADE (i.e., form-and-color-and-depth) theory (for details, see Grossberg, 1994, 1997). Within FACADE there are three main subsystems, the boundary contour system (BCS), the feature contour system (FCS), and the object recognition system (ORS). The BCS forms orientation sensitive and contrast polarity insensitive representations of edge information. This system operates through interactions between short-range competitive and long-range cooperative boundary formation processes at a number of spatial scales and depths to create an ordered representation in depth of all possible boundaries in an image. Although perceptually invisible within the BCS, these boundary representations interact with the ORS and FCS, with FCS processes filling in perceived surface properties (e.g., brightness, color, and texture). Thus, the form, color, and depth of contours and surfaces are only visible through interactions between the BCS and the filling-in processes of the FCS. FACADE properties have been used to explain the perception of phantoms, including the figure/ ground reversability of the illusion with respect to the IA (i.e., with a black IA) and their in-phase appearance (Grossberg, 1994). Long-range cooperative processing at larger scales during the perception of phantoms may explain why phantoms can appear across IA heights much larger than those producing cohesive GI (Zaidi, 1989). FACADE accounts for the lack of phantoms with a gray IA by competition between boundary and surface representations across different depth planes associated with the inducing grating and IA, respectively, as well as ORSBCS interaction's determining which BCS representations are filled in by the FCS (Grossberg, 1997). The surface characteristics of the gray IA block completion of the inducing grating modally, in front of it (e.g., as phantoms). At the same time ORS-BCS interactions allow for the inducing grating to be completed amodally, behind the IA. Although a gray IA effectively eliminates phantoms because of the competition between boundary and surface information, it does allow for local brightnesscontrast effects to appear as surface characterstics within the IA (i.e., GI). ORS influences must certainly contribute to the perception of both phenomena as evidenced by their occurrence in both aligned and misaligned orientations. It may be that ORS influences are less, or at least different, during the perception of GI compared with that of phantoms. The perception of GI might be modelled as a shift toward relatively smaller scale, shorter-range BCS mechanisms' processing local contrast information. Thus, 
the shift in perception between relatively global in-phase phantoms and relatively local out-of-phase GI may be accounted for by specific shifts in the types of interactions occuring between the ORS, the BCS, and the FCS.

Numerous models of perceptual processing emphasize parallel processing of different aspects of a visual scene, wherein global and local aspects are processed by separate but interacting streams (Breitmeyer \& Ganz, 1976; Livingstone \& Hubel, 1987; Weisstein, Ozog, \& Szoc, 1975). It seems that the stimuli used to elicit phantoms and GI may provide bistable illusory situations in which both in-phase and out-of-phase gratings may be perceived, depending on whether attention is allocated to the local or global aspects of the display. If this view is correct, it suggests that phantoms observed with black IAs could be mediated by the $m$-cell stream (Lennie, 1980), whereas phantoms on a gray IA and a GI on either gray or black IAs are mediated by the $p$-cell stream. Although this hypothesis differs from a FACADE account, it might be tested by modifying the displays to favor one or the other stream. The addition of movement to the displays might be expected to yield a preponderance of phantom visibility with low GI visibility, whereas the increase of the spatial frequency of displays and the reduction of the height of the IA might eliminate phantom visibility and enhance GI visibility. Previous studies employing such manipulationshave not measured both phantoms and GI concurrently.

\section{REFERENCES}

BreIt MEYER, B. G., \& GANZ, L. (1976). Implications of sustained transient channels for theories of pattern masking, saccadic suppression, and information processing. Psychological Review, 83, 1-36.

Brown, J. M. (1985). Phantom contour completion: A figure/ground approach. Unpublished doctoral dissertation, State University of New York, Buffalo.

Brown, J. M., \& Weisstein, N. (1985). Flickering phantoms: A figure/ ground approach. Proceedings and Abstracts of the Eastern Psychological Society, 57, 38.

Brown, J. M., \& Weisstein, N. (1988). A phantom context effect: Visual phantoms enhance target visibility. Perception \& Psychophysics, 43, 53-56.

Brown, J. M., \& Weisstein, N. (1991). Conflicting figure-ground and depth information reduces moving phantom visibility. Perception, 20, 155-165.

Foley, J. M., \& McCourt, M. E. (1985). Visual grating induction. Journal of the Optical Society of America A, 2, 1220-1230.
Genter, C. R., II, \& Weisstein, N. (1981). Flickering phantoms: A motion illusion without motion. Vision Research, 21, 963-966.

GrossberG, S. (1994). 3-D vision and figure-ground separation by visual cortex. Perception \& Psychophysics, 55, 48-121.

Grossberg, S. (1997). Cortical dynamics of 3-D figure-ground perception of 2-D pictures. Psychological Review, 104, 618-658.

GyobA, J. (1983). Stationary phantoms: A completion effect without motion and flicker. Vision Research, 23, 205-211.

Gy овA, J. (1994a). Disappearance of stationary visual phantoms under high luminant or equiluminant inducing gratings. Vision Research, 34, 1001-1005.

Gy овA, J. (1994b). The visual phantom illusion under oblique inducing gratings. Japanese Psychological Research, 36, 182-187.

Howell, D. C. (1987). Statistical methods for psychology. Boston: Duxbury Press.

Kellman, P. J., \& Shipley, T. F. (1991). A theory of visual interpolation in object perception. Cognitive Psychology, 23, 141-221.

LENNIE, P. (1980). Parallel visual pathways: A review. Vision Research, 20, 561-594.

Livingstone, M. S., \& Hubel, D. H. (1987). Psychophysical evidence for separate channels for the perception of form, color, movement, and depth. Journal of Neuroscience, 7, 3461-3468.

Maguire, W., \& Brown, J. M. (1987). The current state of research into visual phantoms. In S. Petry \& G. E. Meyer (Eds.), The perception of illusory contours (pp. 213-219). New York: Springer-Verlag.

May, J. G., Brown, J. M., \& Roberts, S. (1999). Afterimages, grating induction and illusory phantoms. Vision Research, 39, 3025-3031.

McCourt, M. E. (1982). A spatial frequency dependent grating induction effect. Vision Research, 22, 119-134.

McCourt, M. E. (1990). Disappearance of grating induction at scotopic luminance. Vision Research, 30, 431-437.

McCourt, M. E. (1994). Grating induction: A new explanation for stationary phantom gratings. Vision Research, 34, 1609-1617.

Nakayama, K., He, J. J., \& Shimojo, S. (1995). Visual surface representation: A critical link between lower-level and higher-level vision. In S. M. Kosslyn \& D. N. Osherson (Eds.), Visual cognition: An invitation to cognitive science: Vol. 2 (2nd ed., pp. 1-70). Cambridge, MA: MIT Press.

SAKURAI, K., \& Gyoba, J. (1985). Optimal inspection area luminance for seeing stationary visual phantoms. Vision Research, 25, 1735-1740.

Tynan, P., \& Sekuler, R. (1975). Moving visual phantoms: A new contour completion effect. Science, 188, 951-952.

Weisstein, N., Maguire, W., \& Berbaum, K. (1977). A phantom motion aftereffect. Science, 189, 955-958.

Weisstein, N., Ozog, G., \& Szoc, G. (1975). A comparison and elaboration of two models of metacontrast. Psychological Review, 82, 321-328.

ZAIDI, Q. (1989). Local and distal factors in visual grating induction. Vision Research, 29, 691-697.

(Manuscript received September 14, 1999; revision accepted for publication May 23, 2000.) 